\title{
Le Cabinet G. P. Vieusseux, entreprise commerciale devenue institut culturel, entre tradition et modernité
}

\section{Gloria Manghetti et Pierre Michel}

Traducteur : Claire Pedotti

\section{(2) OpenEdition}

\section{Journals}

Édition électronique

URL : http://journals.openedition.org/rbnu/1356

DOI : $10.4000 /$ rbnu. 1356

ISSN : 2679-6104

\section{Éditeur}

Bibliothèque nationale et universitaire de Strasbourg

\section{Édition imprimée}

Date de publication : 1 novembre 2015

Pagination : $42-49$

ISBN : 9782859230616

ISSN : 2109-2761

\section{Référence électronique}

Gloria Manghetti et Pierre Michel, « Le Cabinet G. P. Vieusseux, entreprise commerciale devenue institut culturel, entre tradition et modernité », La Revue de la BNU [En ligne], 12 | 2015, mis en ligne le 01 mars 2020, consulté le 14 décembre 2020. URL : http://journals.openedition.org/rbnu/1356 ; DOI https://doi.org/10.4000/rbnu.1356

\section{(c) (i) (2)(2)}

La Revue de la BNU est mise à disposition selon les termes de la Licence Creative Commons Attribution - Pas d'Utilisation Commerciale - Partage dans les Mêmes Conditions 4.0 International. 


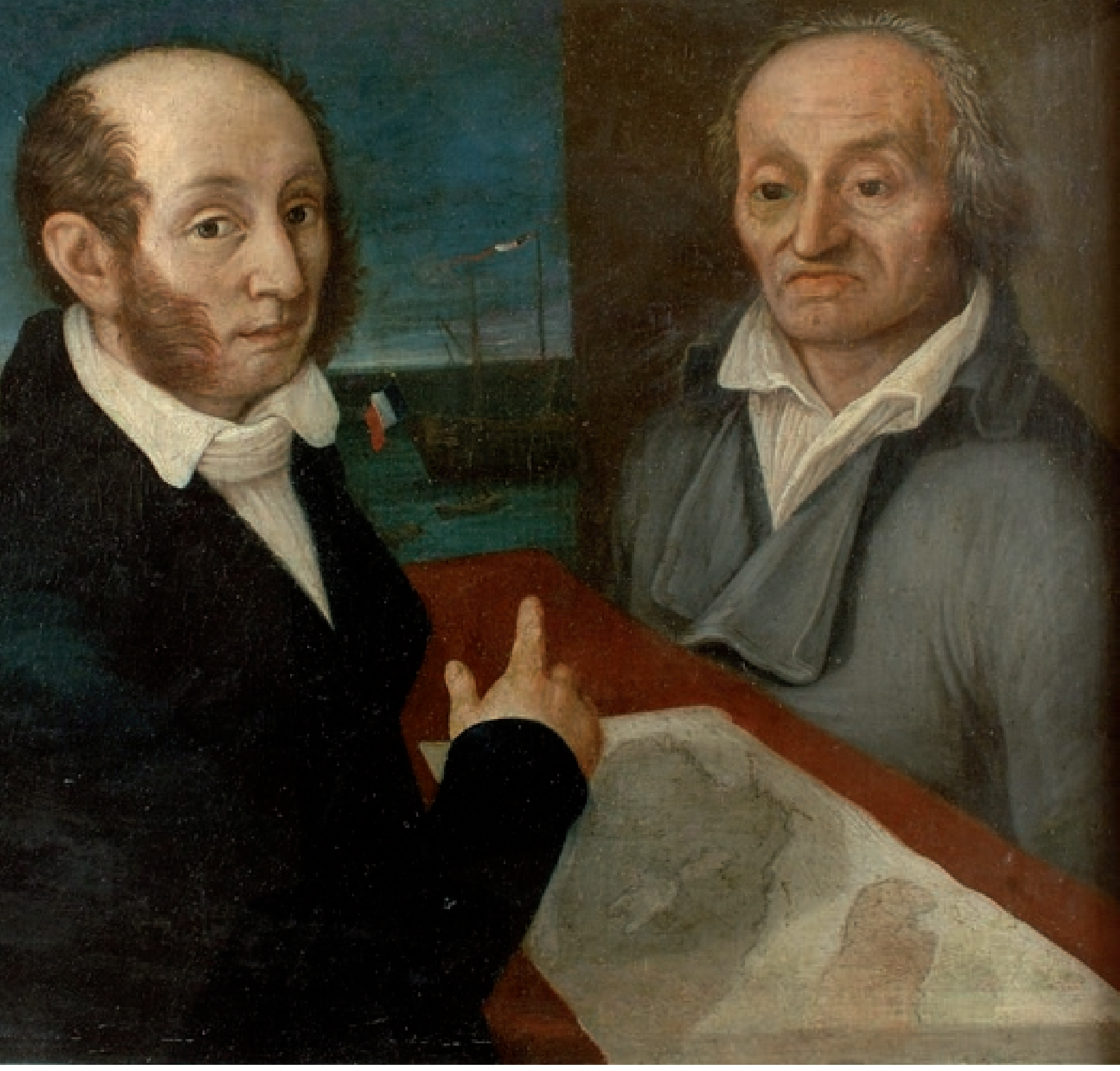

Giovan Pietro Vieusseux et son père Pierre

(anonyme ; huile sur toile, début du $19^{\mathrm{e}}$ siècle ; coll. Cabinet Vieusseux) 


\section{Le Cabinet G. P. Vieusseux,}

\section{entreprise commerciale devenue institut culturel, entre tradition et modernité}

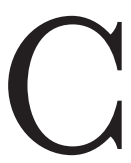

'est le 25 janvier 1820 qu'a eu lieu, au Palais Buondelmonti, piazza Santa Trinita à Florence, l'inauguration du Cabinet scientifique et littéraire G. P. Vieusseux, conçu et fondé par un commerçant d'origine genevoise, né à Oneglia en 1779. Celui-ci, imprégné de la culture des Lumières, avait sillonné toute l'Europe pendant une vingtaine d'années pour son négoce ; ses voyages l'avaient emmené jusqu'à Odessa, à Constantinople et en Grèce ${ }^{1}$. Grâce à ces périples, Giovan Pietro Vieusseux était entré en contact avec les nombreux " cabinets littéraires ", " circulating libraries ", "Leihbibliotheken " qui parsemaient le territoire européen et qui, avec leur structure organisationnelle fondée sur le versement d'une cotisation associative donnant accès aux livres ou aux revues, servirent de modèle au futur " établissement " florentin. Dans le manifeste d'ouverture du 9 décembre 1819, Vieusseux annonçait que les associés auraient à leur disposition : " $1^{\circ}$ Tous les périodiques, journaux et gazettes publiés dans les principales villes italiennes ; $2^{\circ}$ Les périodiques, journaux et gazettes français les plus considérés ; $3^{\circ}$ Certains des meilleurs périodiques, journaux et gazettes anglais et allemands ; $4^{\circ}$ Des cartes géographiques, des dictionnaires et autres ouvrages à consulter ; $5^{\circ}$ Tout le nécessaire pour écrire ". Il indiquait en outre les horaires d'ouverture - tous les jours de l'année de 8 à 23 heures - ainsi que les tarifs - 90 paoli pour un an, 60 pour six mois, 40 pour trois mois, 20 pour un mois, 10 pour une semaine, 2 pour un jour. Peu après (1822), à côté du cabinet de lecture, équipé d'une bibliothèque de consultation, Vieusseux créa la bibliothèque de prêt qui comprenait des ouvrages destinés à l'emprunt à domicile. Il s'agissait surtout de textes contemporains et de nouveautés, principalement dans les domaines de la littérature et de disciplines telles que l'histoire, la géographie, les récits de voyage, les sciences et l'économie. Le rôle de Vieusseux et de son Cabinet scientifique et littéraire s'est alors précisé dans divers champs où se déploya une activité multiforme : des initiatives commerciales et éducatives à celles visant au renouvellement de la culture scientifique et technique, des lettres et des arts, du soutien concret à la circulation des idées à la diffusion de l'édition, initiatives toutes vouées à de grands idéaux civils et politiques. On en veut pour exemple son ferme engagement dans le monde de l'édition, avec la naissance d'une série de revues, de l'Antologia fondée en 1821 au Giornale Agrario Toscano et à la Guida dell'Educatore dirigée par Raffaele Lambruschini, jusqu'à l'Archivio storico italiano, lancé en 1842 et toujours en activité $^{2}$. En effet, le projet ambitieux de Vieusseux portait en son sein tout un ensemble, vaste et complexe, de connotations : par exemple, les affres du Risorgimento italien et les potentialités de la dimension européenne, leitmotiv de la réflexion visionnaire de l'ancien négociant ${ }^{3}$. À cette perspective culturelle contribua incontestablement l'extraordinaire capacité du directeur à réunir autour de lui des intellectuels tels que Gino Capponi, Cosimo Ridolfi, Raffaello Lambruschini, Pietro Giordani, Vincenzo Salvagnoli, Niccolò Tommaseo, qui se rencontraient au Palais Buondelmonti pour débattre de problèmes politiques, littéraires et pédagogiques et de questions scientifiques ou agronomiques. Les soirées en honneur de Giacomo Leopardi et d'Alessandro Manzoni sont restées dans les mémoires. Le 3 septembre 1827 , ils se rencontrèrent au Cabinet Vieusseux, lieu de sociabilité pour de nombreux étrangers qui séjournaient à Florence, dont un grand nombre de noms célèbres : Schopenhauer, 
Stendhal, Fenimore Cooper, Berlioz, Liszt ou Dostoïesvki ${ }^{4}$. Après 1848, le visage du Cabinet florentin se transforma progressivement : il perdit, avec les aspects politiques, son importante dimension de facteur agrégateur, en développant d'autres fonctions dont, surtout, celle du prêt, bien documentée par les registres tenus dès le début des années cinquante pour gérer les services de la bibliothèque de prêt.

Quand, le 28 avril 1863, Vieusseux s'éteignit à l'âge de 84 ans au Palais Buondelmonti, la centralité de son œuvre devint évidente dans une Florence qui, dans les années du Risorgimento, avait dû commencer à concilier l'héritage de son grand passé avec les exigences de la modernités. Sur la pierre tombale du cimetière protestant dit " des Anglais ", ses amis et admirateurs voulurent rappeler ses " nombreux bienfaits pour la civilisation italienne ". N'oublions pas non plus le désarroi qui saisit le cercle rassemblé autour de l'infatigable père fondateur : " Avec la mort de Vieusseux, toute la belle compagnie était dispersée, finie ; et ceux, peu nombreux, qui restaient, se survivaient désormais à eux-mêmes ${ }^{6}$.

Après la disparition de Giovan Pietro, une fois l'unité de l'Italie accomplie et avec les changements du cadre socio-politique, le Cabinet fut dirigé par le neveu du fondateur, Eugenio Vieusseux, qui s'occupa du déménagement en 1873 au Palais Feroni ${ }^{7}$ et intensifia l'activité de la bibliothèque de prêt, en faisant l'acquisition de nouveautés littéraires en provenance d'Italie, de France, d'Angleterre, d'Allemagne et de Russie. Le succès de la bibliothèque, riche d'œuvres en langue originale et dans de grandes traductions, se prolongea jusqu'au siècle suivant, qui enregistra une croissance constante des abonnements. En 1892, la direction passa au fils d'Eugenio, Carlo Vieusseux, dont le nom est lié au déménagement de 1898 au $\mathrm{n}^{\circ} 5$ de la via dei Vecchietti, toujours dans le centre de Florence, mais surtout à la cession, en 1919, du Cabinet et de la bibliothèque au Credito Italiano, qui devint aussi propriétaire du bâtiment qui jouxtait le siège de sa filiale dans la ville ${ }^{8}$. Mais seulement deux ans plus tard, à cause de difficultés de gestion, le Credito céda ce qu'on appelait " le précieux embarras " à la Ville de Florence, qui dut donc lui trouver de nouveaux locaux et en redéfinir le statut juridique. Situé en 1923 près du Palagio di Parte Guelfa, le Cabinet devint personne morale par un décret royal de $1925^{9}$, avec la nomination d'un conseil d'administration présidé par le Podestà - alors Paolo Emilio Pavolini - et la rédaction de statuts restés essentiellement inchangés jusqu'à aujourd'hui, lesquels prévoient à l'article 1 : " L'organisme a pour but de perpétuer l'existence et l'activité du Cabinet scientifique et littéraire G. P. Vieusseux, fondé en mai 1819 , en le maintenant dans ses nobles traditions et en développant ses fonctions en matière de culture et d'étude $"{ }^{10}$. C'est ainsi qu'a été configurée une institution qui, une fois perdue sa nature originelle d'entreprise privée, continuait à avoir une autonomie importante sous la gestion financière de la Ville, laquelle avait dès lors l'obligation statutaire d'en maintenir l'activité. C'est au Conseil, composé de représentants de la Ville, de l'Université et de l'Istituto di Scienze Sociali 'C. Alfieri', qu'incombait aussi la tâche de nommer le directeur de l'Institut, fonctions confiées entre 1925 et 1938 à deux jeunes hommes de lettres : Bonaventura Tecchi (1925-1929) et Eugenio Montale (1929-1938). Tous deux durent faire face à des années extrêmement difficiles, marquées par la diminution sensible des abonnés étrangers. En particulier Montale, confronté à la grave crise financière qui le contraignit à parfois avancer ses fonds personnels, demanda à plusieurs reprises l'augmentation de la contribution municipale et des subventions exceptionnelles pour le Cabinet Vieusseux qui, comme l'écrivait le directeur le 22 novembre 1935 dans un mémoire adressé au préfet de Florence, était alors « la collection la plus riche en Italie pour les livres étrangers modernes et la plus grande bibliothèque de prêt d'Europe " ${ }^{11}$. Ses demandes restèrent toutefois lettre morte et, le $1^{\text {er }}$ décembre 1938 , le Conseil, réuni en l'absence de Montale, décida de relever celuici de ses fonctions de directeur au prétexte qu'il n'était pas inscrit au parti fasciste. On décida, dans ce contexte, de développer les fonctions politiques de l'Institut, avec comme première mesure le déménagement de celui-ci au Palais Strozzi, récemment restauré, qui aurait dû devenir le centre de l'activité intellectuelle, culturelle et touristique de Florence. À cette fin, dans la perspective du déménagement du nouveau siège inauguré le 17 novembre 1940, on nomma à la direction de la bibliothèque un fonctionnaire municipal, Rodolfo Ciullini, lequel, au cours de l'année 1939 (sous la présidence de Jacopo Mazzei qui avait succédé à Paolo Venerosi Pesciolini), effectua un inventaire approfondi de tout le patrimoine des livres de la bibliothèque de prêt, qui comptait plus de 125000 volumes ${ }^{12}$.

En mai 1941, quelques mois après l'installation du Vieusseux au Palais Strozzi, le conseil d'administration, sur proposition du ministère de l'Éducation nationale, nomma directeur Alessandro Bonsanti, en contact avec le Cabinet depuis les années vingt ${ }^{13}$. L'œuvre de Bonsanti, écrivain raffiné et infatigable opérateur culturel, qui tint les rênes de l'Institut jusqu'en 1980, était mue par la 


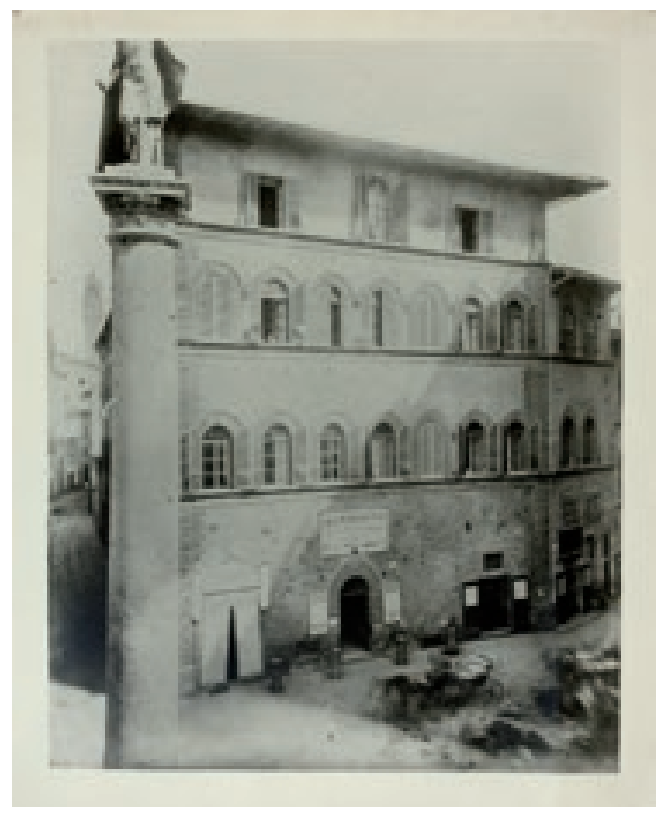

Le Palais Buondelmonti, piazza Santa Trinita à Florence (coll. Cabinet Vieusseux)

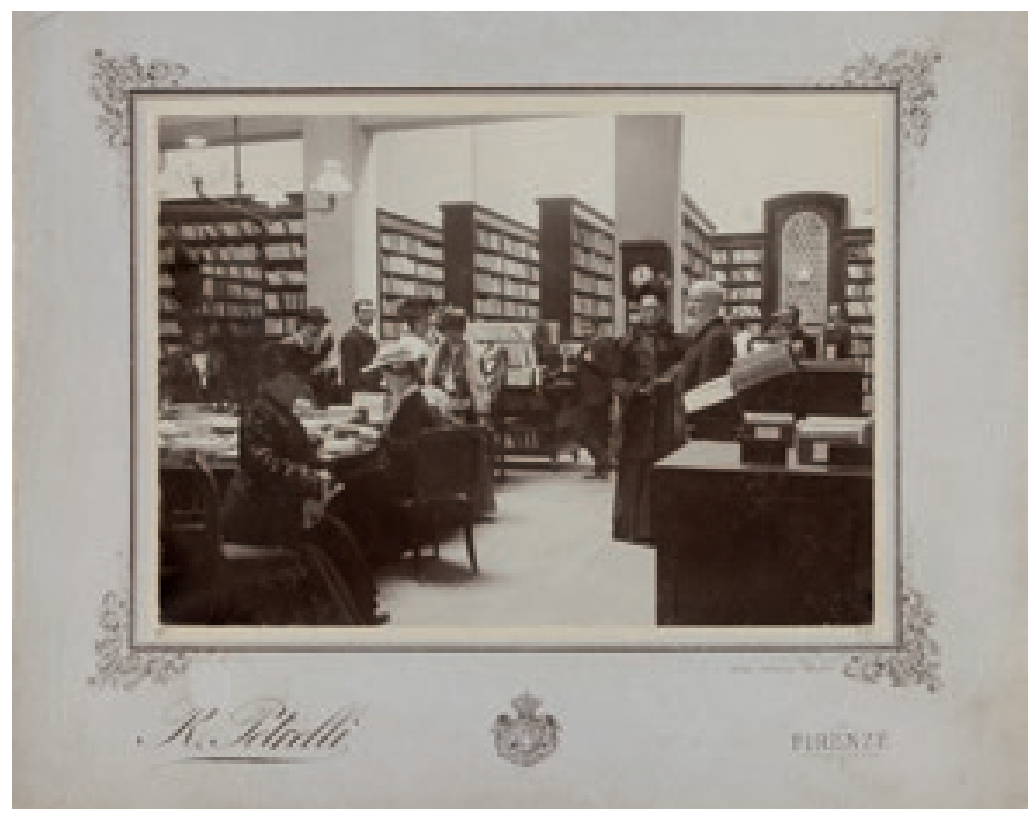

Salle du Cabinet Vieusseux, via dei Vecchietti (coll. Cabinet Vieusseux) volonté de continuer d'accroître l'« influence culturelle " du Vieusseux, "pour la rendre de moins en moins régionale et de plus en plus nationale $"{ }^{14}$. Sous sa direction qui dut, en 1966, faire face aux dommages dramatiques causés au patrimoine par des inondations ${ }^{15}$, le cabinet de lecture et l'extraordinaire bibliothèque de prêt se sont caractérisés par un intérêt de plus en plus actif et dynamique porté à la recherche et à la valorisation du vaste patrimoine documentaire conservé des $19^{\mathrm{e}}$ et $20^{\mathrm{e}}$ siècles. Cette politique, commencée dès le début des années quarante, ne réussit à se concrétiser que beaucoup plus tard avec l'augmentation progressive des initiatives culturelles, le lancement de l'impression, en 1966, d'une revue qui interrogeait ce patrimoine et ses références évocatrices, l'Antologia Vieusseux, mais surtout avec la création du Centro Romantico et de l'Archivio Contemporaneo, conçus comme des lieux de spécialisation de haute culture ${ }^{16}$.

En particulier, le conseil d'administration du Cabinet décida le 27 juin 1973 de créer le Centro Romantico afin de " promouvoir les études sur cette époque culturelle et d'en collecter toute la documentation possible $"{ }^{17}$. Dès lors, ce secteur de l'Institut, en partant du patrimoine relatif aux années du fondateur, s'est consacré à des recherches et des initiatives sur la civilisation européenne du $19^{e}$ siècle, notamment par l'acquisition et la diffusion des connaissances, ainsi que par la comparaison des expériences qui caractérisèrent l'" établissement " de Giovan Pietro. Ouvert à l'interdisciplinarité et aux parcours novateurs dans la tradition de Vieusseux et d'un $19^{\mathrm{e}}$ siècle plus troublé et fécond qui mena à l'unité de l'Italie, le Centro Romantico a élaboré des outils d'accès à un matériel qui devait être appréhendé dans sa totalité pour être vraiment parlant et saisir sur le vif les attentes et les comportements d'une époque ainsi que l'action quotidienne du père fondateur pour la circulation des idées dans l'Europe de son temps. Dans ce contexte, nombreux et variés furent les projets, les occasions de rencontre, les publications concernant les multiples interlocuteurs épistolaires, le réseau des correspondants libraires, la circulation des revues, la documentation sur les voyages et les progrès des sciences, le lectorat, l'actualisation des questions sociales et économiques, l'intérêt pour les " étrangers ", jusqu'à l'élargissement des activités à des problématiques contemporaines comme la médiation entre les cultures ${ }^{18}$.

L'Archivio Contemporaneo, créé le 17 octobre 1975 et qui porte aujourd'hui le nom de Bonsanti, son concepteur et fondateur, fut destiné spécialement à la " sauvegarde " et à la " conservation " de la mémoire du $20^{e}$ siècle. L'idée initiale, conçue en des temps absolument non suspects, se modifia petit à petit et se précisa grâce à l'instinct sûr 


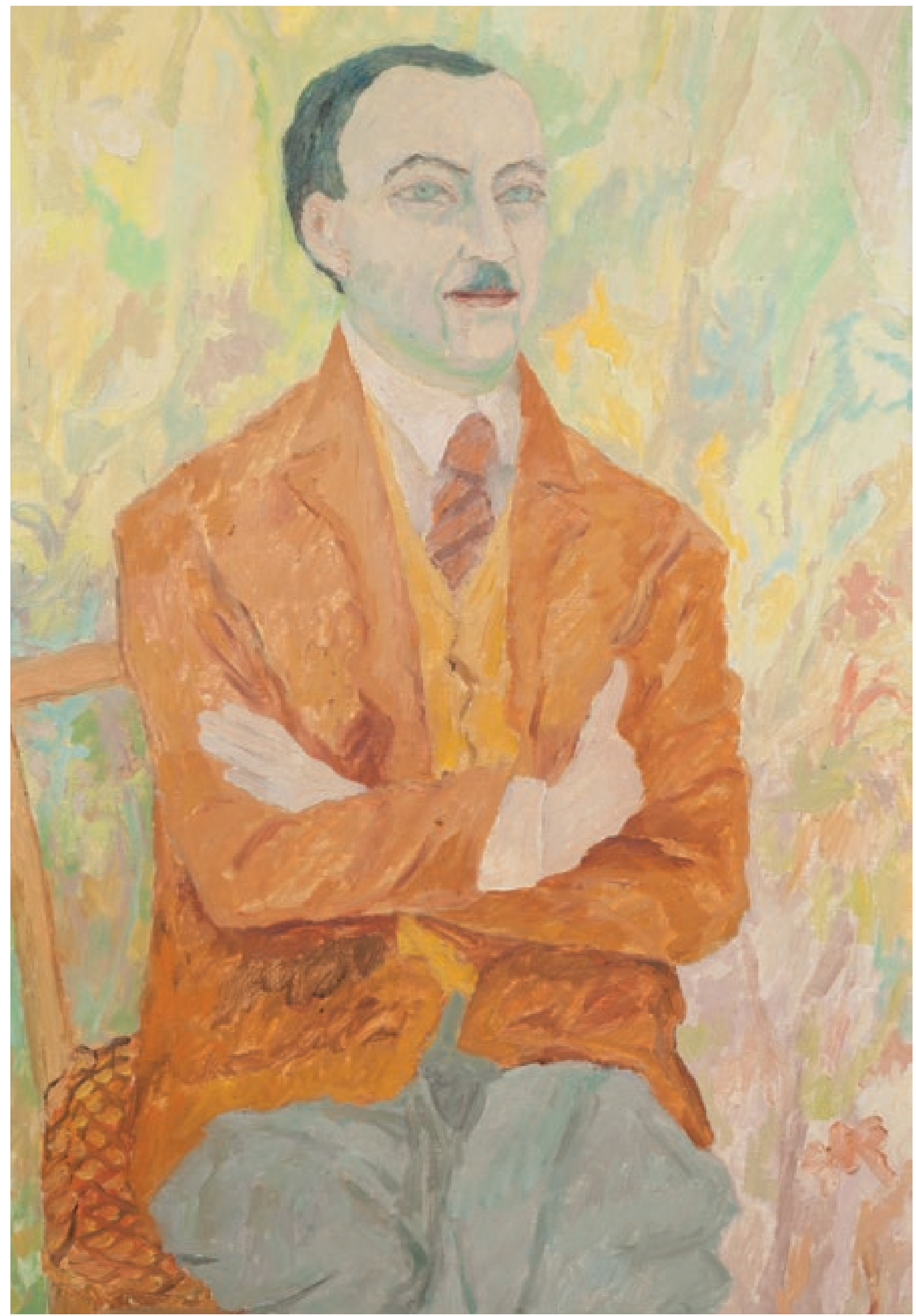

Alessandro Bonsanti peint par Adriana Pincherle (huile sur toile, 1967 ; Archivio Contemporaneo, fonds A. Pincherle) 


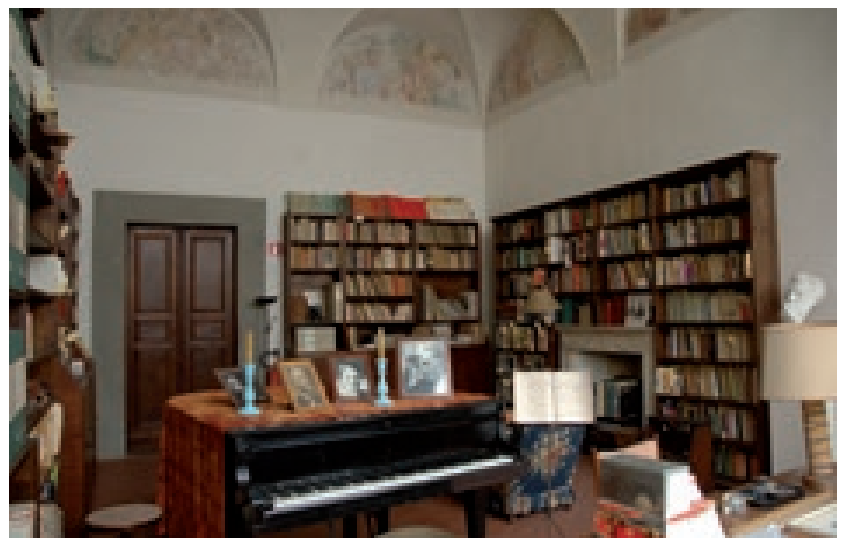

Palais Corsini Suarez, salle du Fonds L. Dallapiccola

du connaisseur qu'était Bonsanti et à son dense réseau d'amitiés dans le monde des lettres, qui l'amenèrent à imaginer la création, dans le cadre de l'Institut et aux côtés de la bibliothèque, d'un nouvel outil de conservation et de connaissance de la culture italienne. L'Archivio Contemporaneo, qui prit bientôt la forme d'un "Institut dans l'Institut " ${ }^{19}$, fut naturellement aussi le fruit d'une volonté plus générale dont Bonsanti eut le mérite de s'approprier les raisons profondes, pour élaborer un outil plus adapté à l'étude des documents contemporains en les préservant de la loi irrésistible de la consommation, avec l'idée que l'histoire littéraire du $20^{e}$ siècle avait elle aussi droit à ses archives. La réponse à cette initiative fut immédiate, si bien que les locaux du Palais Strozzi réservés au Cabinet Vieusseux s'avérèrent insuffisants pour contenir la masse des documents récupérés, lesquels trouvèrent, à la fin de 1979, un emplacement plus adapté dans les salles évocatrices du Palais Corsini Suarez, palais du $14^{e}$ siècle situé à Florence de l'autre côté de l'Arno. L'Archivio où, dans la mesure du possible, la dimension privée de l'acte créateur a été restituée avec un mobilier original dans des espaces bien définis, a vite acquis une spécificité qui le distingue de l'ensemble des instituts créés dans un but de conservation. Dès l'arrivée des premiers documents, souvent remis à Bonsanti en simple gage d'amitié, il se caractérisa en effet par l'éclectisme des disciplines représentées (si, de fait, la littérature italienne en constitue l'ossature principale, on y trouve également des sections liées à des personnalités du monde du cinéma, du théâtre, de la peinture, de l'architecture, de la musique, de la photographie, de l'édition et de la politique), et donc par la multiplicité des matériels conservés (lettres, manuscrits, photographies, coupures de presse, partitions musicales, œuvres d'art, dessins architecturaux, livres, objets et divers souvenirs). Ces documents, laissés là sous forme de dépôts, de prêts, de donations ou de legs testamentaires, font l'objet d'un suivi permanent, confiés aux soins et à l'expérience éprouvée de l'Archivio Contemporaneo. On compte aujourd'hui plus de 150 fonds, souvent enrichis de

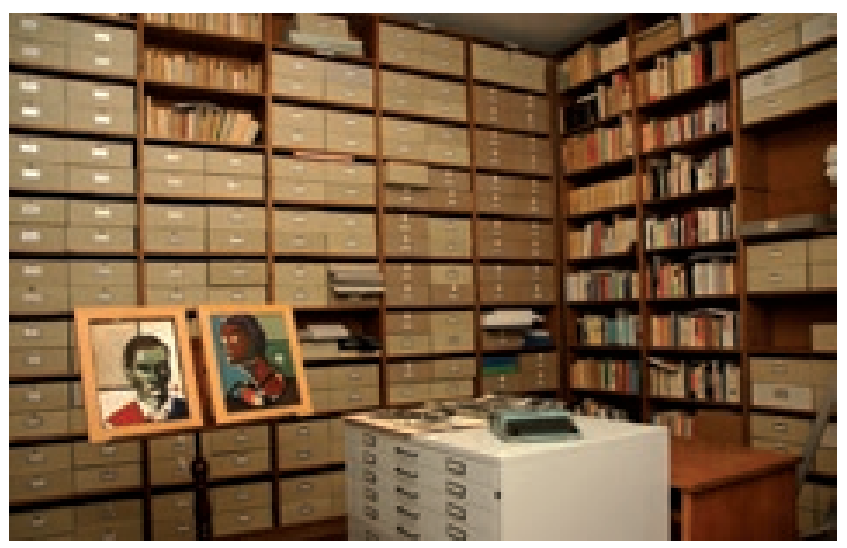

Palais Corsini Suarez, salle du Fonds P. P. Pasolini

bibliothèques personnelles, qui, une fois ordonnés et inventoriés, sont mis à la disposition du public selon des règles précises visant à protéger ce précieux patrimoine. Parmi les nombreux fonds documentaires, citons à titre d'exemple les archives de Giorgio Caproni, d'Emilio Cecchi, d'Edward Gordon Craig, de Luigi Dallapiccola, de Giacomo Debenedetti, d'Eduardo De Filippo, de Carlo Emilio Gadda, de Mario Luzi, d'Angiolo et d'Adolfo Orvieto, de Pier Paolo Pasolini, de Vasco Pratolini, d'Alberto Savinio, de Mario Tobino, de Federigo Tozzi, de Giuseppe Ungaretti... ${ }^{20}$ Dans son histoire postérieure, le Cabinet Vieusseux, qui a vu se succéder à la tête de l'Institut Marino Raicich (1980-1984), Geno Pampaloni (1984-1985), Luigi Crocetti (1985-1986), Paolo Bagnoli (1987-1994), Enzo Siciliano (1995-200o), Giovanni Gozzini (2000-2007), jusqu'à la direction actuelle, a poursuivi une réflexion organique sur lui-même, en consacrant des études et des actions à ses propres origines et en promouvant des projets conçus pour rendre accessible une mémoire précieuse entre passé et présent. Il suit par là un itinéraire caractérisé par une activité intense, multiforme et exigeante, marquée d'emblée par des desseins souvent ambitieux et menés sur divers fronts, dans une perspective culturelle où " cohabitent l'ancien et le nouveau " ${ }^{21}$, dans la conviction que c'est précisément sur le terrain d'une interdisciplinarité vivante que peut se jouer l'avenir de l'Institut. Les quelques propos, mesurés et incisifs, consacrés par Bonsanti dans les années soixante-dix au Centro Romantico, dans un programme global recouvrant aussi son autre créature, l'Archivio Contemporaneo, trouvent aujourd'hui une nouvelle concrétisation en nous rappelant que l'on peut " être d'avant-garde tout en regardant, non pas en avant, mais en arrière ", en oscillant " entre passé et présent, ou plutôt entre passé et avenir " et en liant, selon une grande tradition, " la culture à la politique, la "littérature" à la vie ${ }^{22}$.

\section{Gloria Manghetti \\ (traduction Claire Pedotti)}




\section{Le fonds Dallapiccola du Gabinetto G. P. Vieusseux de Florence (Archivio Contemporaneo "A. Bonsanti ")}

En tant que musicologue, j’ai pu travailler à plusieurs reprises au Gabinetto Vieusseux et consulter les nombreux documents du fonds concernant le compositeur Luigi Dallapiccola (1904-1975), l'une des figures importantes de la musique italienne après Puccini. Ce fonds est d'une grande richesse ; il permet d'appréhender sous différents angles la démarche et les activités de ce compositeur avant, pendant et après la Seconde Guerre mondiale. Il est tout à fait comparable aux archives Schoenberg (Venise) ou au fonds Webern conservé à la Fondation Paul Sacher de Bâle, deux compositeurs dont Dallapiccola était pour ainsi dire le disciple tout en ayant choisi une voie différente de celle des trois grands représentants de la Seconde école de Vienne.

En 1995, à l'occasion d'un colloque sur le compositeur florentin, la musicologue Mila De Santis a fait paraître un ouvrage/catalogue du fonds Luigi Dallapiccola ${ }^{23}$ qui correspondait à la fin du classement et de l'inventaire des documents rassemblés à l'Archivio Contemporaneo. Ce livre permet donc de savoir précisément en quoi consiste ce fonds ; le sommaire distingue les "musiques" (partitions autographes, livrets, etc.), les écrits (manuscrits, manuscrits d'autres auteurs concernant Dallapiccola, traductions italiennes de textes divers par Dallapiccola), les écrits sur le compositeur et finalement un index des correspondants (privés et institutions) avec les dates des pièces correspondantes.

Le travail de documentation effectué par Mila De Santis est extrêmement intéressant pour la culture italienne en général, et il est intéressant de citer un extrait de son introduction à l'ouvrage :

" Le fonds Dallapiccola comprend une collection fournie d'autographes musicaux, la correspondance, les écrits (édités et inédits), la bibliographie critique, la collection des programmes des concerts au cours desquels des œuvres de Dallapiccola ont été jouées et les critiques les concernant (documentation quasi complète jusqu'à la disparition du compositeur, mais - on le comprendra - plus lacunaire ensuite), les documents (lieux, programmes, articles, etc.) concernant l'activité didactique et concertiste, les enregistrements discographiques et les conférences. S'y ajoute une petite collection de programmes d'opéras et de concerts auxquels Dallapiccola put assister, dans les années vingt et le début des années trente, à la Staatsoper de Berlin, à
l'Augusteo de Rome, à la Gesellschaft der Musikfreunde et au Konzerthaus de Vienne ; toute la collection des médailles, des prix, des diplômes et des décorations qui lui ont été conférés tout au long de sa carrière ; un riche dossier iconographique, comprenant certains portraits à l'huile et des centaines de photographies (personnelles et de scène). Le fonds comprend aussi les journaux (53 carnets et deux cahiers de notes), actuellement scellés et non consultables, selon les dispositions testamentaires, pendant les cinquante années postérieures à la mort du compositeur. À la disposition des chercheurs, mentionnons également les partitions et les réductions imprimées de ses pièces musicales, une magnétothèque, enrichie de temps à autre par les interprètes, et une petite collection de matériels audiovisuels, parmi lesquels le documentaire Odysseus, Geburt einer Oper, réalisé par la ZDF à l'occasion de la première de Ulisse à Berlin, le documentaire monographique réalisé par Maryvonne Kendergi pour la télévision canadienne, l'entretien avec Massimo Mila sur Ulisse, repris par la télévision de la Suisse italienne (Lugano) en 1972, ainsi que l'enregistrement de Ulisse au Teatro Regio de Turin (12 décembre 1986)".

Signalons que l'auteure a également publié quelques années plus tard les actes du colloque qui avait lieu au moment de cet achèvement du travail de recensement du fonds Dallapiccola. L'ouvrage est intitulé : Dallapiccola Letture e prospettive. Una monografia a più voci, a cura di Mila De Santis, Lucca, Ricordi LIM, 1997.

\section{Pierre Michel}

(traduction du texte italien par Claire Pedotti)

\section{Notes}

1 - Pour l'histoire de l'Institut florentin, voir Il Vieusseux. Storia di un Gabinetto di lettura 1819-2003. Cronologia Saggi Testimonianze, sous la direction de Laura Desideri, Florence, Polistampa, 2004, où figure aussi une bibliographie spécifique.

2 - L'Archivio storico italiano, qui a eu plusieurs séries, est la revue historique italienne la plus ancienne encore publiée et l'une des plus vieilles du monde ; elle est aujourd'hui publiée sous les auspices de la Deputazione di Storia Patria per la Toscana.

3 - Voir Giovan Pietro Vieusseux. Pensare l'Italia guardando all'Europa (actes de colloque, Florence, 27-29 juin 2011), Florence, Olschki, 2013.

4 - On retrouve des traces importantes de la présence de ces personnalités, animant la ville symbole du cosmopolitisme du $19^{\mathrm{e}}$ siècle, dans les 23 grands volumes du Libro dei soci (Registre des associés). On renvoie à cet égard aux projets importants pilotés par Laura Desideri : de la numérisation systématique, à consulter sur le site de l'Institut (www. vieusseux.fi.it), à la transcription intégrale des abonnés. À L. Desideri, en collaboration avec Monica Pacini, on doit aussi la réalisation d'un produit multimédia, constamment mis à jour, qui suit l'évolution historique du Cabinet de lecture dans ses différents locaux : Il Vieusseux 
\& Firenze, disponible en ligne à l'adresse www.vieusseux.it/multimedia/ vieusseux-firenze-2012/gvp_vieusseux-firenze-ridotto.html. Voir Monica Pacini, "Viaggiatori-lettori a Firenze prima e dopo l'Unità ", in Libri e lettori verso l'Italia unita: dalle fonti del Gabinetto Vieusseux 1820-1870, actes de la journée d'étude, Florence, Palais Strozzi, 22 avril 2010, in Antologia Vieusseux, n. s., a. XVII, $n^{\circ}$ 49-50, janvier-août 2011, p. 59-84.

En ce qui concerne les relations entre Leopardi et Vieusseux, nous nous limiterons à évoquer Leopardi nel carteggio Vieusseux. Opinioni e giudizi de contemporanei (1823-1837), sous la direction d'Elisabetta Benucci, Laura Melosi, Daniela Pulci, 2 vol., Florence, Olschki, 2001.

5 - En 1863, après plus de quarante ans d'activité intense, le Cabinet pouvait se vanter d'un patrimoine d'environ 300 périodiques, 270 ouvrages de consultation et de plus de 11 ooo volumes de la bibliothèque de prêt, dont $46,8 \%$ en français, $24,7 \%$ en anglais, $24,6 \%$ en italien et un peu moins de $2 \%$ en allemand. Les abonnés au Cabinet, inscrits dans le registre des associés dès le premier jour, sont surtout des voyageurs étrangers d'origines diverses : Anglais, Américains, Français, Allemands, Russes, Polonais, Suisses, etc. Les quarante premières années, le Cabinet comptait 30000 associations.

6 - Raffele Ciampini, Giovan Pietro Vieusseux. I suoi viaggi, $i$ suoi giornali, i suoi amici, Turin, Einaudi, 1953, p. 459.

7 - Voir Laura Desideri, " Il Gabinetto Vieusseux nelle sale di Palazzo Feroni (1873-1898) ", in Un Palazzo e la città, sous la direction de Stefania Ricci et de Riccardo Spinelli, Milan, Skira, 2015, p. 210-227 ; voir aussi Laura Desideri, " La biblioteca di Eugenio Vieusseux con il testo inedito Della biblioteca (1882) ", in Antologia Vieusseux, n. s., a. XXI, n 62, mai-août 2015, p. 5-26.

8 - L'opération s'éleva à 800 ooo lires pour l'immeuble et 400 ooo lires pour la bibliothèque.

9 - Voir Gazzetta ufficiale, $\mathrm{n}^{\circ}$ 275, 26 novembre 1925, p. 4679.

10 - L'article 5 des statuts établissait la composition du Conseil, lequel devait comprendre sept membres : « le Podestà de Florence et toute autre personne déléguée par celui-ci, trois personnes nommées par le conseil municipal, deux nommées par le Sénat académique de la R. Università et une nommée par le Conseil des professeurs du R. Istituto superiore di Scienze Sociali C. Alfieri ». Les statuts actuels ont été approuvés en 1989 (décision du Conseil d'administration de l'Institut $\mathrm{n}^{\circ} 15$ du 6 octobre 1989 ; décision du Conseil régional de la Toscane $\mathrm{n}^{\circ} 134$ du 6 mars 1990), avec modification postérieure de l'article 5 (décision du Conseil d'administration de l'Institut $\mathrm{n}^{\circ} 2$ du 28 avril 2011 ; décision du Conseil municipal de Florence $n^{\circ} 9$ du 21 mars 2011).

11 - Paolo Bagnoli, "Montale e il Gabinetto Vieusseux ", in Il Vieusseux, a. V, n 13, janvier-avril 1992, p. 71.

12 - Les données sont tirées du rapport de Ciullini, Trasporto del Gabinetto Vieusseux in Palazzo Strozzi, à propos duquel on renvoie à Laura Desideri, "Il Gabinetto G. P. Vieusseux ", in Palazzo Strozzi, cinque secoli di arte e cultura, sous la direction de Giorgio Bonsanti, Florence, Nardini Editore, 2005 , p. 116.

13 - Parmi les nombreuses contributions consacrées à la direction de Bonsanti, rappelons les actes du colloque du 20 avril 2004, Alessandro Bonsanti nel centenario della nascita, in Antologia Vieusseux, n. s., a. $\mathrm{X}, \mathrm{n}^{\circ} 30$, septembre-décembre 2004. Voir encore la monographie de l'Antologia Vieusseux, ${ }^{\circ}$ 27, septembre-décembre 2003, qui comprend entre autres la Bibliografia degli scritti di Alessandro Bonsanti, sous la direction de Laura Malatesti, p. 27-16o ; voir aussi L'Archivio Storico del Gabinetto Vieusseux. Inventario, sous la direction de Caterina Del Vivo et de Luisa Di Tolla, Florence, Polistampa, 2011, en particulier les p. 313-402.

14 - Alessandro Bonsanti, " Il romantico Giampietro ", in Oggi, 14 juin 1941.

15 - Environ 90\% du patrimoine des livres conservés dans les sous-sols du Palais Strozzi furent gravement endommagés. Bonsanti décida de créer un centre de récupération et un laboratoire de restauration, fusionnés en 1984 dans une structure unique encore active. Le Service de conservation se consacre aujourd'hui à la sauvegarde et à la restauration des documents (papiers et livres) conservés à l'Institut.
16 - « Dès le début de l'après-guerre, il fut évident que le Cabinet G. P. Vieusseux devait modifier l'orientation principale de sa mission, vouée dans le demi-siècle précédent presque exclusivement à la gestion de la bibliothèque, pour redevenir un institut aux intérêts culturels vastes, dynamiques et modernes [...] Tout comme il était clair que, parmi les initiatives à prendre, la priorité devait être donnée à la publication d'un organe périodique pour les mêmes raisons récurrentes qui avaient poussé Giovan Pietro Vieusseux à éditer l'Antologia " : nous citons là l'Avvertimento - non signé mais de la main de Bonsanti - qui ouvrait le premier cahier de l'Antologia Vieusseux, janvier-mars 1966, p. 1. L'Antologia Vieusseux continua sous la direction de Bonsanti jusqu'au décès de celui-ci le 18 février 1984, puis cessa d'être publiée en 1986. Elle a été reprise, dix ans après, avec une nouvelle série, par Enzo Siciliano.

17 - Parmi la vaste bibliographie sur le Centro Romantico, nous nous limiterons à évoquer le premier numéro - entièrement consacré au Centro - de la nouvelle série de l'Antologia Vieusseux et en particulier la contribution de Maurizio Bossi, "Intorno all'impresa di Vieusseux. Una premessa ", in Antologia Vieusseux, n. s., a. I, $n^{\circ} 1$, janvier-avril 1995, p. 11-16. Il est impossible de choisir parmi les nombreux projets soutenus, mais il nous plaît de mentionner le travail important sur la correspondance de Vieusseux et le récent ouvrage de Letizia Pagliai, Repertorio dei corrispondenti di Giovan Pietro Vieusseux dai carteggi in archivi e biblioteche di Firenze (1795-1863), Florence, Olschki, 2011.

18 - Dans cette perspective, aux côtés du Centro Romantico, citons la naissance du programme 'Vieusseux-Asia', qui a suivi l'acquisition en 1997 de la bibliothèque orientale et de la photothèque de Fosco Maraini, qui voulait relier de manière cohérente son œuvre à l'idée, chère à Vieusseux, de mise en contact de la culture italienne avec les autres cultures.

19 - Ces propos de Bonsanti sont extraits d'une lettre adressée le 16 avril 1980 à la Direction générale du Bureau central pour le livre et les instituts culturels du ministère des Biens culturels et environnementaux. S’agissant de la spécificité de l'Archivio Contemporaneo, Bonsanti précisait aussi que " cet "Institut dans l'Institut" " présentait " quelque chose de nouveau (au moins pour l'Italie) dans la manière d'organiser et de cataloguer les fonds ". La " définition structurelle de l'Archivio Contemporaneo " fut décidée le $1^{\text {er }}$ décembre 1979 (décision $n^{\circ} 11 / 79$ ), pendant la réunion du Conseil d'administration lors de laquelle Bonsanti présenta sa démission des fonctions de directeur de l'Institut et où il fut nommé conservateur de l'Archivio, poste qu'il occupa jusqu'à sa mort. Par ailleurs, rappelons qu'un an auparavant, en 1983, Bonsanti était devenu maire de Florence. Voir aussi Gabriele Turi, " Marino Raicich direttore del Gabinetto Vieusseux ", in Marino Raicich intellettuale di frontiera, Florence, Olschki, 2000, p. 107-127 (en particulier le paragraphe "Un direttore dimezzato ", p. 112-118). Dans la vaste bibliographie sur l'Archivio Contemporaneo, nous citerons : L'Archivio Contemporaneo "Alessandro Bonsanti" del Gabinetto G. P. Vieusseux, sous la direction de Caterina Del Vivo et de Gloria Manghetti, Florence, Associazione nazionale archivistica italiana. Sezione toscana, Quaderni di Archimeetings, 2004 ; Gloria Manghetti, " Dal Gabinetto G. P. Vieusseux: l'Archivio Contemporaneo "A. Bonsanti" tra tradizione e modernità ", in Clara Borrelli, Elena Candela, Angelo R. Pupino (sous la direction de), Memoria della modernità. Archivi ideali e archivi reali, actes du XIII congrès international de la MOD, tome I, Pise, ETS, 2013, p. 89-105.

20 - Sur le site de l'Institut, on consultera la liste complète des fonds, avec de brèves indications sur la personne qui en est à l'origine, les contenus et les instruments de recherche, ainsi que les inventaires en ligne.

21 - Voir Marino Raicich, "Bonsanti, il Vieusseux, la letteratura contemporanea. Presentazione ", in Antologia Vieusseux, a. XX, $\mathrm{n}^{\circ} 3$, juillet-septembre 1984 , p. 56, cahier consacré à Bonsanti peu de mois après sa disparition en février 1984 .

22 - Les citations sont tirées de : Alessandro Bonsanti, Centro romantico, in Antologia Vieusseux, a. XIV, $\mathrm{n}^{\circ}$ 3, juillet-septembre 1979, p. 1 ; Alessandro Bonsanti, "Romantico, in oggi ", in Antologia Vieusseux, a. IX, $\mathrm{n}^{\circ} 3$, juillet-septembre 1974 , p. 1 .

23- Fondo Luigi Dallapiccola - Autografi, scritti a stampa, bibliografia critica con un elenco dei corrispondenti, sous la direction de Mila De Santis, Florence, 1995, Inventari 5, Gabinetto G. P. Vieusseux de Florence (Archivio Contemporaneo « A. Bonsanti »). 